\title{
Active filtering applied to radiographic images unfolded by the Richardson-Lucy algorithm
}

\author{
Almeida, G. L. ${ }^{1}$ \\ Instituto de Engenharia Nuclear - Comissão Nacional de Energia Nuclear \\ P. O. Box 68550, ZIP 21945-970 Cidade Universitária, Rio de Janeiro, Brazil \\ E-mail: gevaldo@ien.gov.br \\ Silvani, M. I. \\ Instituto de Engenharia Nuclear - Comissão Nacional de Energia Nuclear \\ P. O. Box 68550, ZIP 21945-970 Cidade Universitária, Rio de Janeiro, Brazil \\ E-mail: msouza@ien.gov.br
}

\section{Lopes R. T.}

Universidade Federal do Rio de Janeiro - Laboratorio de Instrumentação Nuclear

P. O. Box 68509, ZIP 21945-970 Cidade Universitária, Rio de Janeiro, Brazil

E-mail: ricardo@lin.ufrj.br

Degradation of images caused by systematic uncertainties can be reduced when one knows the features of the spoiling agent. Typical uncertainties of this kind arise in radiographic images due to the non-zero resolution of the detector used to acquire them, and from the non-punctual character of the source employed in the acquisition, or from the beam divergence when extended sources are used. Both features blur the image, which, instead of a single point exhibits a spot with a vanishing edge, reproducing hence the point spread function - PSF of the system. Once this spoiling function is known, an inverse problem approach, involving inversion of matrices, can then be used to retrieve the original image. As these matrices are generally illconditioned, due to statistical fluctuation and truncation errors, iterative procedures should be applied, such as the Richardson-Lucy algorithm. This algorithm has been applied in this work to unfold radiographic images acquired by transmission of thermal neutrons and gamma-rays. After this procedure, the resulting images undergo an active filtering which fairly improves their final quality at a negligible cost in terms of processing time. The filter ruling the process is based on the matrix of the correction factors for the last iteration of the unfolding procedure. Synthetic images degraded with a known PSF, which have undergone the same treatment, have been used as a test to evaluate the soundness of the developed active filtering procedure. The unfolding and filtering algorithms, as well as the softwares to plot all images and generate the synthetic ones, have been written in Fortran 90 language.

XXXIV edition of the Brazilian Workshop on Nuclear Physics,

Foz de Iguaçu, Parana state, Brasil

5-10 June 2011

${ }^{1}$ Almeida, G. L. 


\section{Introduction}

Radiographic image acquisition systems are affected by several spoiling agents which degrade the quality of the final image. Roughly, they could be classified into two groups: random agents such as scattering and statistical dispersion and those of systematic character such as detector resolution and beam divergence for instance. Random uncertainties require some kind of smoothing or filtration to mitigate them, a somewhat risky approach, for high spatial frequencies eventually belonging to the signal itself are regarded as noise and eliminated too.

The systematic uncertainties can in principle be eliminated without risks of affecting the true signal if one knows their features and behavior. In practice this ideal scenario does not occur, for random uncertainties of some kind always arise together with the systematic ones. As each pixel looses part of its original intensity, which is spread over its surrounding neighbors, affecting them as well, the whole original image is degraded.

Yet, as the degradation process has a systematic character, the information is not lost but solely concealed. Assuming for instance an image acquired by a system with a known and fully characterized point spread function - PSF, it would be possible to assemble a linear system where the independent vector was comprised by the intensity of the individual pixels in the observed image and the coefficient matrix comprised by numbers deduced from the PSF.

After this approach, an original image comprised by $n \times m$ pixels would require a coefficient matrix with the size $(n \times m)^{2}$ and a $n \times m$ independent vector. One can promptly figure out that even a modest $640 \times 480$ pixels image would require more than $94 \mathrm{~Gb}$ of allocation memory. Although this number is feasible for the current state-of-art of storage devices, it is not for programs dealing with the inversion of matrices as required to solve linear systems. Furthermore, a program capable to deal with it would certainly require a very long time to invert such a huge matrix.

Disregarding these constraints, and assuming that no noise infected the image during its acquisition, even so, random uncertainties would emerge as arithmetic truncation errors in the coefficient matrix making it ill conditioned which could produce a solution vector full of outliers and negative values. The unfeasibility to apply this elegant method lead to the development of alternative approaches such the iterative Richardson-Lucy algorithm, henceforth referred in this paper as RL.

This algorithm has been applied in this work to improve radiographic images acquired by transmission of thermal neutrons and gamma-rays using an exponential curve to compose both sides of the PSF used as unfolding function. Its better width - expressing the resolution of acquisition system - has been experimentally determined and used to obtain a set of images by successive iterations after the RL approach. A high number of iterations, nevertheless, does not necessarily assure a good image. Beyond a certain number, besides the additional computational costs, degradation may occur. As from this point on, no further improvement can be achieved with the RL approach, this work proposes an extension of this algorithm through the application 
of an active filtering to the unfolded image, which fairly improves its final quality at a negligible processing time cost.

The filter is based on the matrix of the correction factors for the last iteration of the unfolding procedure. Synthetic images degraded with a known PSF, which have undergone the same treatment, have been used as test to evaluate the soundness of the developed active filtering procedure. The unfolding and filtering algorithms have been incorporated to a Fortran 90 program.

\section{Methodology}

The main theme of this work is the filtering of images previously improved by unfolding with the RL algorithm. However, prior to this treatment, several methods, techniques and procedures have to be employed to prepare the image for this filtering including the RL deconvolution. These activities are then grouped into three sections: Data Setup, RL Deconvolution and Active Filtering itself, as follows.

\subsection{Data Setup}

All radiographic images processed in this work have been acquired with imaging plates, and hence, they were black \& white and already in digital format. Their original sizes, as arising from the imaging plate reader, were $8040 \times 4096$ pixels in tiff format. Prior to their input in the Fortran program, they were rescaled and transformed into jpg format by using the ImageJ software which furnishes as well the related optical density matrix $d(x, y)$ in the txt format. This rescaling, performed in order to reduce the matrix sizes and concomitantly to spare processing time, was chosen so that the final image could match the current 1440 x 900 pixels monitors.

\subsection{Richardson-Lucy Deconvolution}

The well-known Richardson-Lucy [1, 2] algorithm is an iterative procedure for retrieving a latent image blurred by a known PSF. So, the pixel intensity in the observed image can then expressed as,

$$
d_{i j}=\sum_{k=i-\Delta k}^{i+\Delta k} \sum_{l=j-\Delta l}^{j+\Delta l} p_{i j k l} u_{k l}
$$

where:

$d_{i j}=$ Pixel intensity observed at location $(i, j)$.

$u_{k l}=$ Pixel intensity in the latent (unknown) image at location $(k, l)$.

$p_{i j k l}=$ PSF: contribution of the pixel $u_{k l}$ of the latent image to the pixel $d_{i j}$ in the observed image.

$\Delta k=$ Range of the PSF along the $k$-direction.

$\Delta l=$ Range of the PSF along the $l$-direction. 
The above mentioned ranges refer to the maximum absolute distances $|k-i|$ and $|j-l|$ where the PSF centered at $(k, l)$ still contributes significantly to the intensity of the pixel at $(i, j)$. Actually, the surrounding pixels giving the same contribution (assuming they had the same intensity) would be located in a circle around the reference pixel, as expected from an isotropic PSF. However, as this approach would increase the processing time to determine the coefficient matrix, the fixed ranges above specified have been adopted in this work. Their values have been chosen as a certain number of PSF widths, assuring thus an automatic fit to them.

The basic idea behind the RL algorithm is to calculate the most likely $u_{k l}$ given the observed $d_{i j}$ and known $p_{i k l j}$. This leads to an equation for $u_{k l}$ which can be solved iteratively as follows,

$$
u_{k l}^{t+1}=u_{k l}^{t} \sum_{i=k-\Delta k}^{k+\Delta k} \sum_{j=l-\Delta l}^{l+\Delta l} \frac{d_{i j}}{C_{i j}} p_{i j k l}
$$

where:

$u_{k l}^{t}=$ Pixel intensity in the unknown image at location $(k, l)$ after the iteration $t$.

$u_{k l}^{t+1}=$ Pixel intensity in the unknown image at location $(k, l)$ after the iteration $t+1$. and,

$$
c_{i j}=\sum_{k=i-\Delta k}^{i+\Delta k} \sum_{l=j-\Delta l}^{j+\Delta l} p_{i j k l} u_{k l}^{t}
$$

As can be seen in eq. (2) the double summation is a factor correcting the pixels intensities along two successive iterations, henceforth in this paper called $f_{k l}^{t}$, where $t$ is the iteration number,

$$
u_{k l}^{t+1}=u_{k l}^{t} \cdot f_{k l}^{t}
$$

From the above formulation, two main requirements emerge: the necessity to know the PSF, except for the blind deconvolution approach [3], and the number of iterations in order to achieve the best results. The PSF, a feature of system employed to acquire the image, is somewhat cumbersome to be fully characterized. Yet, approximate bell-shaped functions, provided that they possess a representative FWHM, can fairly perform the task.

In this work, a PSF decreasing along the distance from its center as an exponential decay,

$$
p\left(x_{0}, x, y_{0}, y\right)=e^{-\left(\frac{2 \cdot \ln 2}{w}\right) \cdot \sqrt{\left(x-x_{0}\right)^{2}+\left(y-y_{0}\right)^{2}} \mid}
$$

where:

$$
\begin{aligned}
p\left(x_{0}, x, y_{0}, y\right)= & \text { Contribution of the pixel located at the PSF center }\left(x_{0}, y_{0}\right) \text { to the pixel } \\
& \text { located at }(x, y) . \\
w= & \text { Full width at half maximum - FWHM. }
\end{aligned}
$$


has been applied as deconvolution function. This function has been chosen because better images have been obtained with it than with the Gaussian type, most likely due to the reducible character of this function.

The best PSF width $w$ has been deduced from the Line Spread Function - LSF after the formalism described in [4]. This LSF has been obtained by the differentiation of the Edge Response Function - ERF, experimentally determined after the approach employed in [5]. Once the best width was obtained, several iterations were carried out, and for each of them, the resulting image was stored for further analysis.

A high number of iterations, however, does not necessarily assure a good image and may even degrade the previously improved ones. Indeed, as observed in this work, beyond a certain number of iterations, besides the additional computational costs, artifacts may appear. From this point on, no further improvement can be achieved with the RL approach, as used in this work. Hence, after the better achievable image was obtained, an active filtering - the actual subject of this work - was applied to it, according to the described procedures as follows.

\subsection{Active Filtering}

From eq. (4) one can promptly figure out that when all the elements of the matrix $f_{k l}^{t}$ reach the unity no further improvements can take place. As this ideal scenario would never occur, a possible figure of merit to evaluate the goodness of the deconvolution, as proposed by Lucy [2], could be the overall deviation of $f_{k l}^{t}$ from the unity expressed as,

$$
\chi_{t}^{2}=\frac{1}{n \cdot m} \sum_{k=1}^{m} \sum_{l=1}^{n}\left[1-f_{k l}^{t}\right]^{2}
$$

where $m$ and $n$ are the number of columns and lines in the image. This criterion, however, seems inappropriate as a tool to decide when to stop the iterations. Indeed, the unfolded radiographic images of an old-fashioned film photo-camera acquired with $412 \mathrm{keV}$ gamma-rays from a ${ }^{198} \mathrm{Au}$ source, as shown in Fig. 1 exhibit a fair improvement until 50 iterations.

Further iterations nevertheless, not only do not improve the image, but even spoil it, as seen by the given example with 1,000 iterations. The parameter $\chi_{t}^{2}$, on the other hand, exhibits a steadily asymptotic decreasing with $t$ in spite of the gradual image degradation. The deconvolution has been carried out with a PSF width of $0.84 \mathrm{~mm}$. 


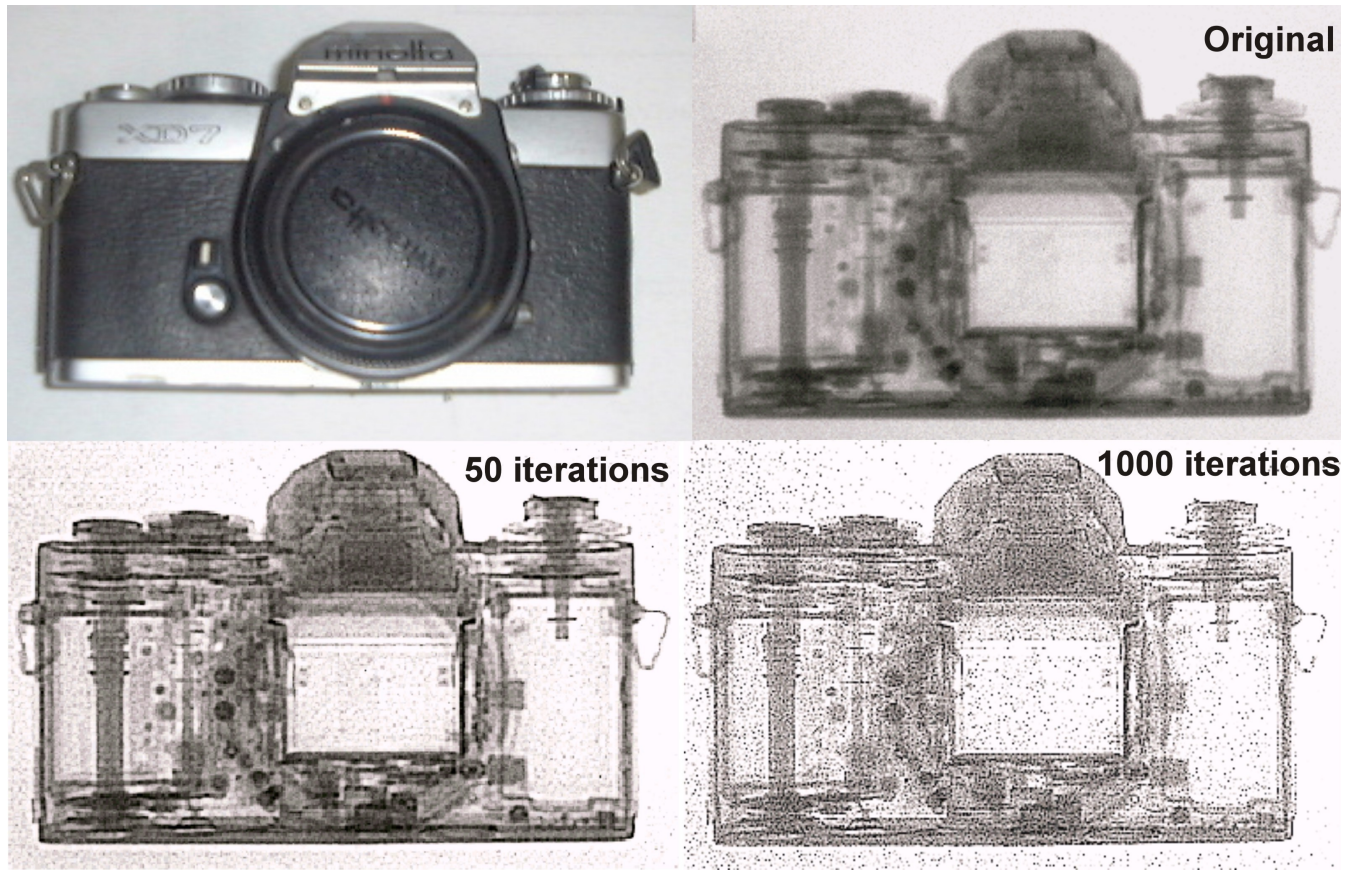

Fig.1 - Original and unfolded radiographs of a film photo-camera acquired with $412 \mathrm{keV}$ gamma-rays and an $\mathrm{x}$-ray sensitive imaging plate. A conventional photo is shown for reference.

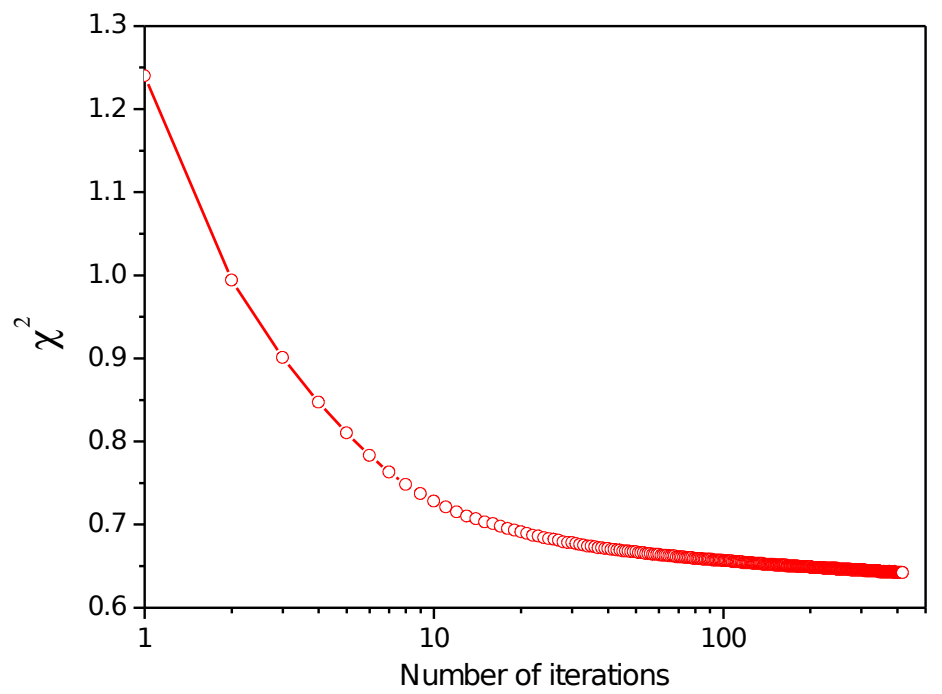

Fig. 2 - Behavior of $\chi^{2}$ against the number of iterations. The image quality does not follow this asymptotic trend, becoming more and more degraded after about 50 iterations. 
A non-zero $\chi^{2}$ means obviously that many values of $f_{k l}^{t}$ deviate from the unity, in spite of the several performed iterations. Values still above and below unity give an intuitive indication that $f_{k l}^{t}$ is trying to correct the next value $u_{k l}^{t+1}$ of $u_{k l}^{t}$. Yet, as the elements of the matrix $f_{k l}^{t}$ are tied to each other through the PSF centered at each pixel, the whole web precludes individual changes. If somehow, the individual values of $f_{\mathrm{kl}}^{t}$ could be changed disregarding their companions and without affecting them, then it would be possible to correct at once the individual values of $u_{k l}^{t}$ by applying a factor which were no longer tied to the whole web, but to the deviation from the unity.

A correction of this nature naturally should not affect the factors $f_{k l}^{t}$ equal to unity. Furthermore, it should be proportional to the deviation from the unity, i.e., factors originally far from the unity should be corrected more vigorously than those near to it. The concept behind this approach is that, if even after many iterations, the factor $f_{k l}^{t}$ is still very low/high, it is because it did not succeed to decrease/increase respectively the value of $u_{k l}^{t+1}$ to a proper level. Hence, the correction should be accelerated.

An algorithm capable to perform this task would act as an active filter, correcting each pixel intensity at once. A possible, but not ultimate, filter of this kind can be expressed as,

$$
\begin{aligned}
& g_{k l}=e^{-p\left(1-f_{k l}^{t}\right)} \\
& u_{k l}^{g}=u_{k l}^{t} \cdot g_{k l}^{t}
\end{aligned}
$$

where:

$f_{k l}^{t}=$ Correction factor matrix of the last iteration.

$u_{k l}^{t}=$ Pixel intensity matrix from the last iteration.

$g_{k l}^{t}=$ New correction factor matrix after the $t^{\text {th }}$ iteration.

$u_{k l}^{g}=$ Final pixel intensity matrix.

$p=$ Filter parameter.

After this approach, the filter is applied only one time to the pixel intensity matrix obtained in the last iteration.

\section{Results and Discussion}

Prior to the application of the filtering to actual unfolded images, its soundness was tested with a synthetic image, generated without any noise and afterwards degraded with a chosen well defined PSF. In spite of the employing of the very same PSF for both degradation and deconvolution it was impossible to recover the original image but only to improve the degraded 
one. As shown in Fig. 2, the unfolded \& filtered image exhibits a slight improvement with regard to the solely unfolded one.

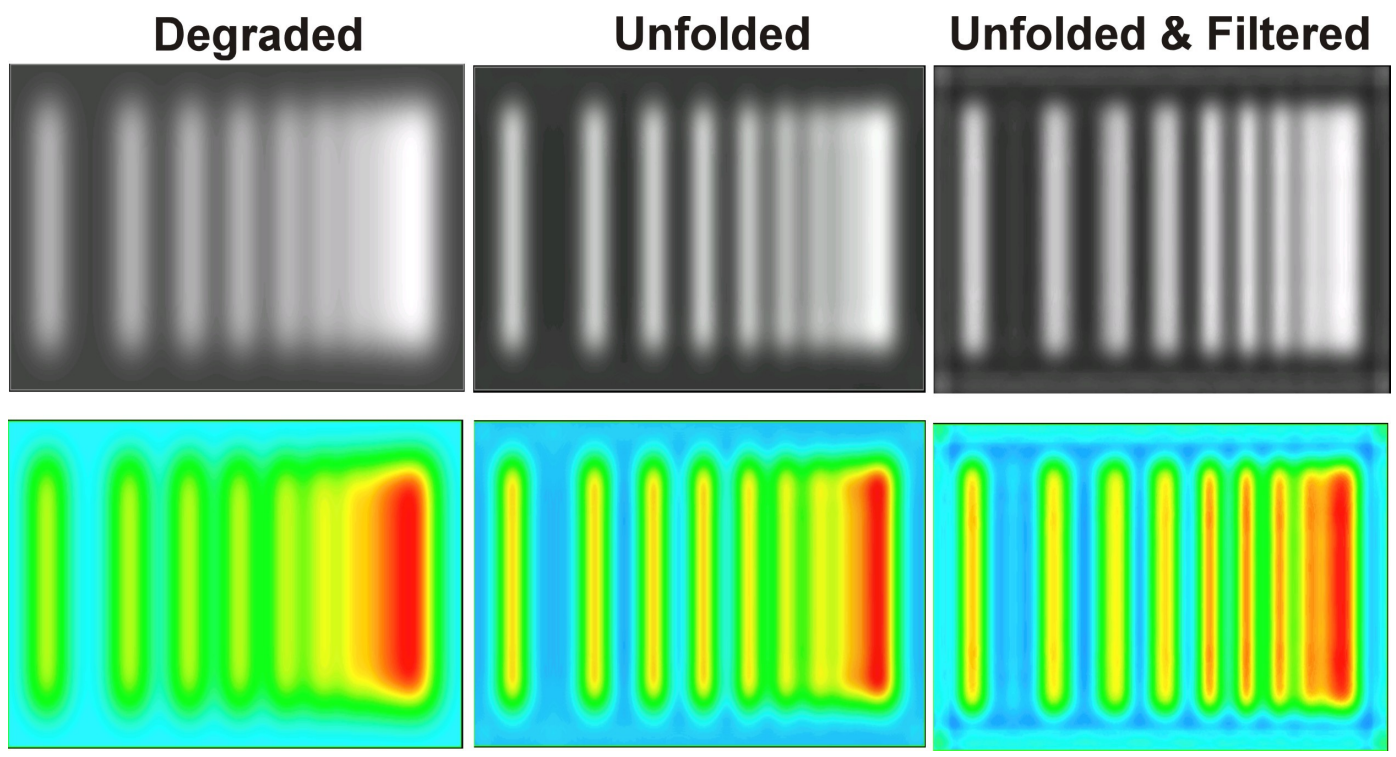

Fig. 2 - Synthetic images which have undergone degradation, unfolding, and unfolding \& filtration. A colored version is shown to facilitate the differentiation between them.

The best parameter $p$ for the filter has been evaluated by trial and visual inspection. This procedure will be discussed later on, in this work, when dealing with actual radiographic images. In order to facilitate the comparison, the black \& white images have been colored by associating the gray scale to a rainbow one.

\subsection{Analyzing the soundness of the filtering algorithm}

Once verified that the filtration of synthetic images yielded reasonable results, the algorithm has been applied to real images acquired with neutron and gamma-ray radiography. Due to its purely intuitive character, the filtering algorithm should undergo some kind of tests to evaluate its soundness. The criteria adopted in this work were its performance for real and different images, its behavior for filters doing just the opposite intended effect, the impact of $p$ upon the quality of the filtered image, and its effect upon $\chi^{2}$ as well.

In order to address the first criterion, the neutron radiographic image of a pocket chronometer has been unfolded and filtered, as formerly done with the gamma radiographic image of a photo-camera. The results depicted in Fig. 3 shows a fair improvement of the unfolded \& filtered image with regard to that solely unfolded as highlighted by the zoomed 
images beneath them. A p-value of 50 has been used for the filtration after a trial and visual inspection approach.
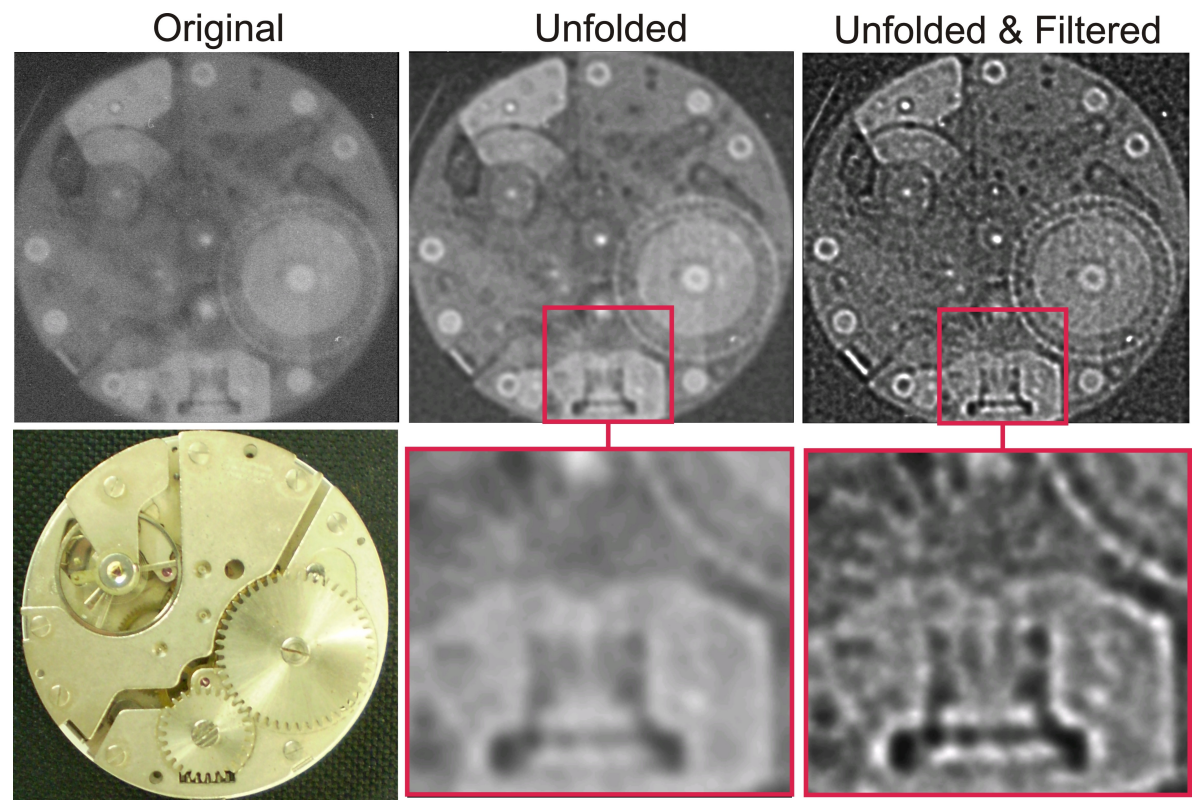

Fig. 3 - Neutron radiographs of a pocket chronometer. The zoomed areas highlight the improvement achieved with the filtering. A conventional photo is also shown for reference.

The effects of two alien filters are shown in Fig. 4. While the proposed filter produced an acceptable image (centre) the other ones yielded final images full of artifacts. The image at the right side is interesting, for it was produced by a filter with a positive parameter. This means that the filter would be trying to increase/reduce the low/high factors $f_{k l}^{t}$ respectively.

Although this seems at first sight to be a possible alternative - the matrix elements $f_{\mathrm{kl}}^{t}$ would be shifted towards the unity - such an assumption does not match with the behavior of the previously applied RL algorithm, which was trying to reduce/increase the low/high factors. Furthermore, the final image ratifies that a positive $p$ is not a good choice. Obviously this outcome alone, does not assure the soundness of the proposed filtering algorithm, but it is a step further towards its acceptance as a reliable tool.

An additional analysis aiming to understand what is going on in the filtering approach is to verify the appearance of the matrix $f_{k l}^{t}$. As it would be difficult and cumbersome to compare the numerical values, they have been converted into a gray scale and into a rainbow scale of optical densities. The results shown in Fig. 5 indicate that the highest deviations from the unity occur at the edges, .i.e., at the highest pixel intensity gradients. This outcome may be most likely caused by the attempts of the deconvolution algorithm to overcome the difficulty to deal with such high gradients.

The situation could be illustrated with the example of a degraded step function exhibiting a Boltzmann-like shape. An algorithm intended to recover the original function would try to 
increase/decrease the values above/below its discontinuity point respectively, keeping nevertheless the values at this point unchanged, i.e., performing the same task as the filter used in this work.

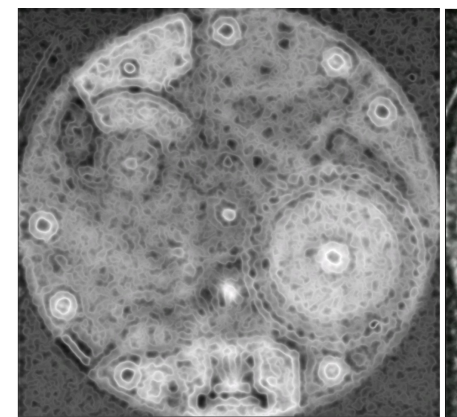

$$
g_{k l}=e^{-p\left|1-f_{k l}^{t}\right|}
$$

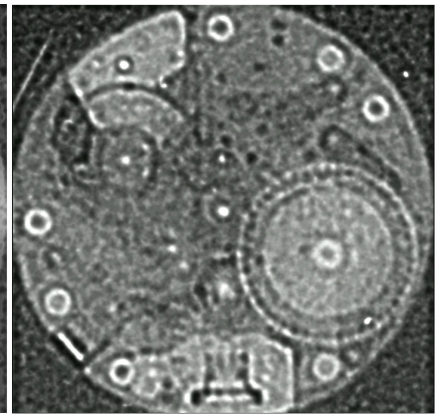

$g_{k l}=e^{-p\left(1-f_{k l}^{t}\right)}$

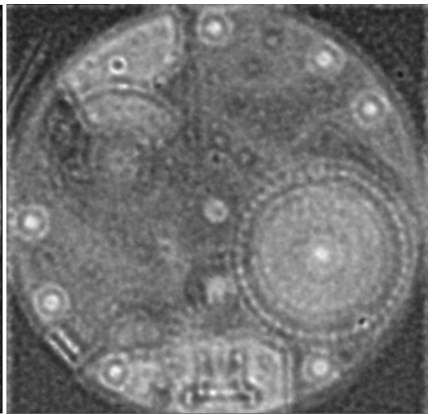

$$
g_{k l}=e^{+p\left(1-f_{k l}^{t}\right)}
$$

Fig.4 - The deconvoluted image treated with the proposed filter (centre) exhibits acceptable quality, while those treated with alien filters (left and right) are full of artifacts.
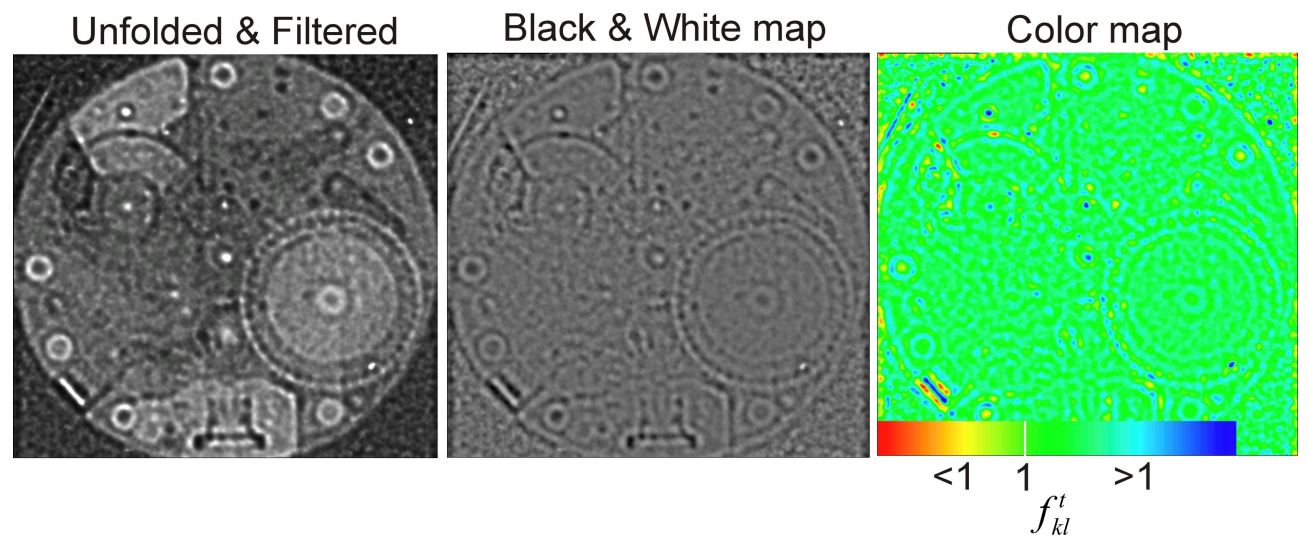

Fig.5 - Map of the correction factors $f_{k l}^{t}$ converted into pixel intensities. As seen in the color map, the highest deviations of $f_{k l}^{t}$ from the unity occur at the edges. The unfolded \& filtered neutron radiograph is also shown for reference.

\subsection{Impact of the filter parameter on the image quality}

The effect of the filter parameter $p$ upon the quality of the final image is an essential aspect for an effective application of the filtering algorithm. This effect is as well, one of the criteria related to the soundness and capability of the filter to improve previously unfolded images. The Fig. 6 shows an unfolded image and three other ones filtered with different $p$-values. 

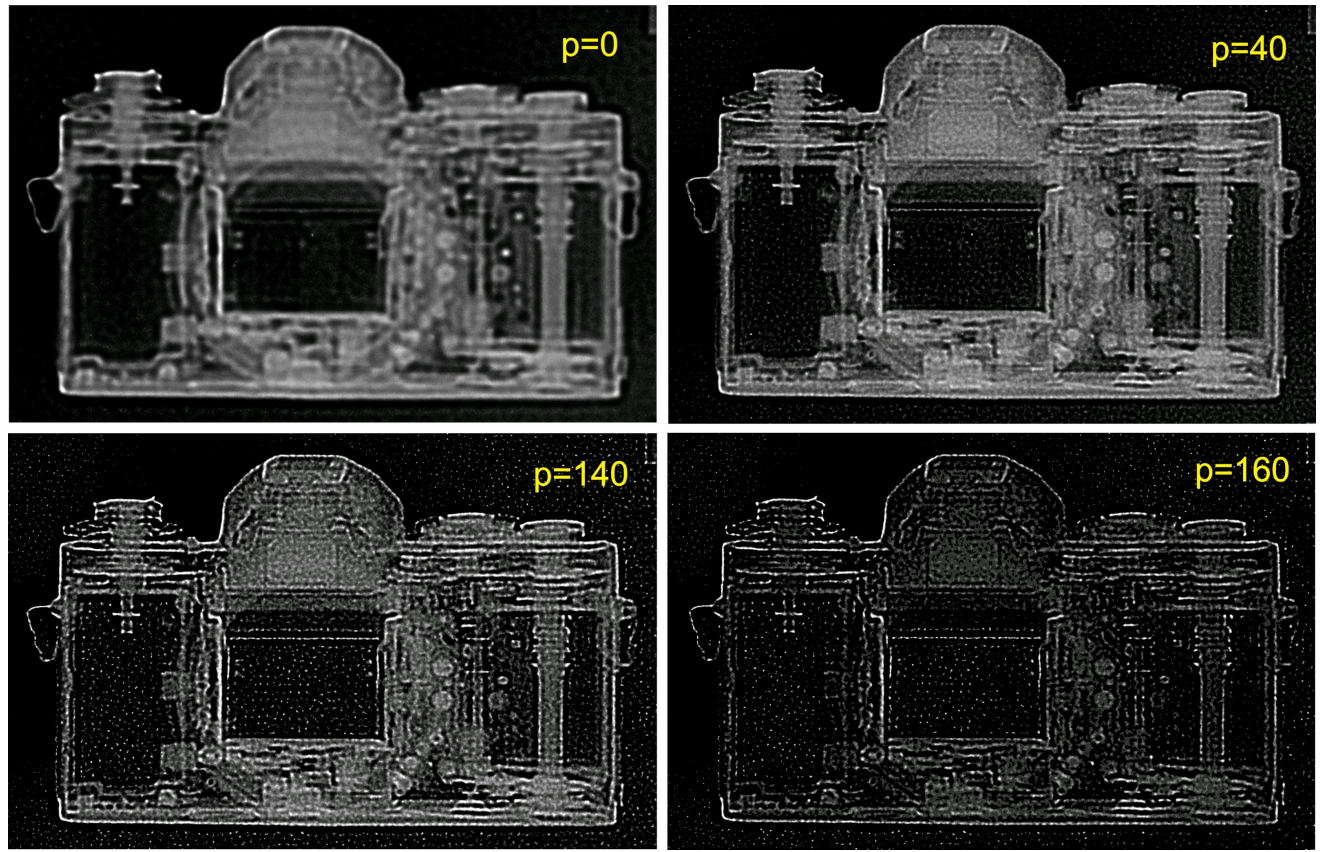

Fig. 6 - Original deconvoluted image (top left) and the related filtered ones with three different $p$-values. The optimal image is obtained with a $p$-value of about 40 . Outside this region the image is not adequately filtered or it is over-filtered with poorer results.

A $p=0$ means no filtration at all. As the filtration with increasing $p$-values proceeds, (always using the same original deconvoluted image), the image is initially improved, but beyond a certain $p$-region it becomes more and more degraded.

The choosing of a proper $p$ by trial and inspection would be a very cumbersome procedure, as it would be necessary to plot all images and compare them. Therefore, the effect of this parameter on $\chi^{2}$ has been analyzed, as an attempt to find a correlation between them and the quality of the filtered image. Results in Fig. 7 show that beyond the inflexion point, where the best image occurs, $\chi^{2}$ grows very quickly, an increasing accompanied by the rapid degradation of the image.

This outcome, besides reducing the subjectiveness of a visual inspection, allows a simpler and faster way to choose $p$, since it is much easier to compare numbers than patterns. Furthermore, it makes possible an automatic determination of $p$ by the computer program carrying out the filtration. 

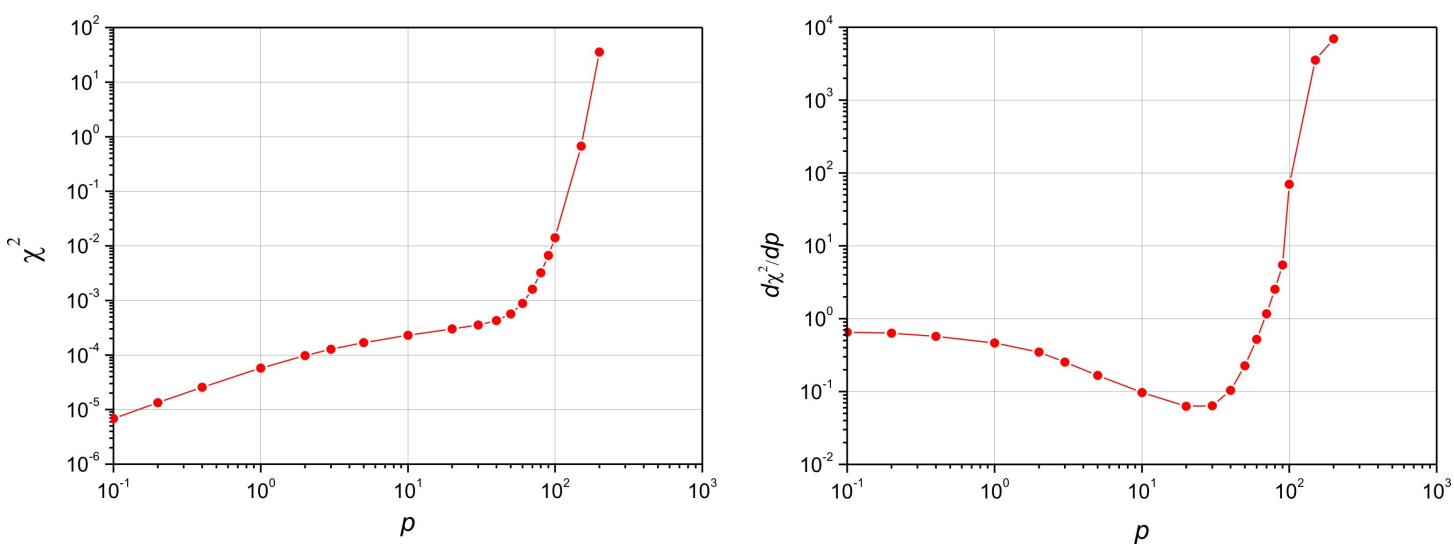

Fig. 7 - Impact of the filter parameter $p$ on $\chi^{2}$. Beyond the inflexion point, better visualized through its $1^{\text {st }}$ derivative, $\chi^{2}$ grows very quickly, a behavior which is reflected as a quick degradation of the related filtered image.

\section{Conclusions}

An active filtering has been applied to radiographic images unfolded by the classical Richardson-Lucy algorithm, which fairly improves their final quality at negligible processing time costs. Synthetic images degraded with a known PSF, have undergone the same treatment, and used as a test to evaluate the soundness and consistence of the filtering procedure. The filter ruling the process employs the matrix of the factors correcting pixel intensities between two subsequent iterations. The idea behind this filtering procedure is that factors still exhibiting signficative deviations from the unity, even after numerous iterations, have been trying to correct the corresponding pixels intensities, but were partially unsuccessful, for they were tied to the whole matrix through the PSF. Within this frame, each factor is corrected, based upon its own deviation from the unity, and upon a common parameter which can be automatically evaluated by the filtering algorithm itself. The unfolding and filtering algorithms, as well as the softwares to plot all images, and generate the synthetic ones, have been written in Fortran 90 language. Although this work addresses only radiographic images, the methodology has a general character, being applicable to any kind of digital images. Previously unfolded, neutron and gamma-ray transmission radiographs of some objects have been filtered with fairly good results. Despite the modest improvement, the additional computational effort for the filtering is negligible, making the cost-benefit quite acceptable.

\section{References}

[1] W. H. Richardson, Bayesian-Based Iterative Method of Image Restoration, Journal of the Optical Society of America, 62 (1) 55, 1972. 
[2] L. B. Lucy, An iterative technique for the rectification of observed distributions, The Astronomical Journal 19 (6) 745, 1974.

[3] D. Kundur, D. Hatzinakos, Blind Image Deconvolution, IEEE Signal Processing Magazine, 13 (6) $61,1996$.

[4] E. W. Marchand, Derivation of the Point Spread Function from the Line Spread Function, Journal of of the Optical Society of America, 54 (916) 7, 1964.

[5] M. I. Silvani, G. L. Almeida, J. D. Rogers, R. T. Lopes, Stereoscopic Radiographic Images with Thermal Neutrons, Nuclear Instruments and Methods in Physics Research A, http://dx.doi.org/10.1016/j.nima.2010.09.051 